\title{
Intercontinental spread of a highly transmissible clone of Pseudomonas cepacia proved by multilocus enzyme electrophoresis and ribotyping
}

WENDY M JOHNSON MD PhD

$\mathrm{T}$ EN YEARS AGO A CANADIAN REPORT DREW ATTENTION TO Pseudomonas cepacia as an emerging pathogen among cystic fibrosis (CF) patients (1). It is now well recognized that $P$ cepacia can be a significant pathogen in some $\mathrm{CF}$ patients with respiratory colonization or infection and may be associated with higher morbidity and mortality $(2,3)$. Although nosocomial infections have been reported and are usually attributed to contaminated aqueous and disinfectant solutions, $P$ cepacia is rarely found in environmental clinical settings. This is in marked contrast to the reservoirs of Pseudomonas aeruginosa, which can include sinks, nebulizing equipment, plastic tubing and other common hospital sites. Available data strongly suggest that the CF patient infected with $P$ cepacia is the major source of infection for other CF patients (4), and there are observations that cross-infection with $P$ cepacia in nosocomial situations occurred in $\mathrm{CF}$ patients in both the United States (5,6) and the United Kingdom (7). Additionally, Govan et al (8) recently concluded that social contact between CF patients and an infected person can pose a significant risk of infection.

To understand better the routes of transmission for $P$ cepacia, laboratory analysis of pulmonary isolates must encompass typing methods that are sensitive, discriminatory and reproducible. Unfortunately, pheno-

National Laboratory for Special Pathogens, Bureau of Microbiology, Laboratory Centre for Disease Control, Ottawa, Ontario

Correspondence and reprints: Dr Wendy M Johnson, Bureau of Microbiology. Laboratory Centre for Disease Control, Tunney's Pasture, Ottawa, Ontario K1A OL2 typic variability has been a hallmark of $P$ cepacia, and methods relying on phenotype do not provide the degree of assurance required to fulfil our epidemiological goals. Genotypic characterization of $P$ cepacia is preferable in that variable expression of phenotype is avoided and, to date, ribotyping has been the most useful and is the current reference 'standard' in view of its reproducibility and discriminatory power (9). None of these methods has adequately assessed the degree of genetic biodiversity among strains and, indeed, the important question of whether some isolates are inherently more virulent than others remains unaddressed.

Two pieces of evidence are highly suggestive that some strains of P cepacia may have enhanced virulence. First, there is a wide variation in prevalence and incidence of $P$ cepacia infection observed among $\mathrm{CF}$ patients in the clinics of North America and Europe, which seems unlikely to be due entirely to different clinical practices. Second, a recent report by Govan et al (8) documents the emergence and spread by social contact of a highly transmissible 'epidemic' strain within and between $\mathrm{CF}$ clinics.

To establish whether particular strains were unique to any geographic area or had a higher prevalence or adverse outcome, we recently analyzed genetic relationships among 83 isolates of $P$ cepacia from widely divergent geographic areas (10). Multilocus enzyme electrophoresis (MLEE) and ribotyping were used to characterize these strains and, although most were isolated from $\mathrm{CF}$ patients, a number from non-CF nosocomial infections and reference environmental sources were included in our analysis for comparative pur- 
poses. Twenty enzyme electrophoretic types (ETS) were determined, of which one clone, ET12, was associated with six of nine ribotype strains said to be geographically representative of the United Kingdom. All of the Ontario isolates tested from $\mathrm{CF}$ patients also belonged to ET12. Clone ET12 was not found among isolates from nosocomial infections in Ontario or environmental strains and was never found in CF isolates from British Columbia, Nova Scotia or a large centre in the eastern United States. Ribotypes of individual isolates did not cluster geographically as did the ET signatures, as noted above, and frequently ribotypes occurred in more than a single ET. Single ETs usually contained several ribotypes unless associated with a point source outbreak. Dendrographic analysis grouped those strains from CF patients, nosocomial outbreaks and environmental sources into separate ET families, and diversity analysis indicated that, with the exception of ET17, CF isolates clustered in unique and closely related ETs different from those from nosocomial and environmental sources.

This study has also shown the potential of MLEE to follow the intercontinental spread of $P$ cepacia strains in CF patients. The United Kingdom 'epidemic' is anecdotally linked to a Canadian summer camp, and our analysis has shown many of the strains in the United Kingdom and Ontario to be of the same electrophoretic type. Additional investigation will be required to establish the initial date and direction of spread of $P$ cepacia (ET12) between the United Kingdom and Ontario. These circumstances suggest that camps undertaken by $\mathrm{CF}$ patients in regions far removed from their own communities may represent a risk for infection with different $P$ cepacia strains. Whether this intercontinental ET12 clone, which now predominates in the United Kingdom and Ontario, has more than normal virulence to $\mathrm{CF}$ patents is uncertain, but it can be assumed to be highly transmissible and infectious.

A statement from the Medical/Scientific Advisory Committee of the Canadian Cystic Fibrosis Foundation concerning the epidemiology of $P$ cepacia in $\mathrm{CF}$ appeared in 1993 in this journal (2). This article summarizes the current state of knowledge and clinical concern about $P$ cepacia pulmonary infections in $\mathrm{CF}$ patients and describes a Canadian initiative to establish a central repository of $P$ cepacia isolates from $\mathrm{CF}$ patients across Canada. This collection, created by voluntary collaboration, will be essential if the many unanswered questions relating to the epidemiology of $P$ cepacia are to be successfully addressed.

Although several genotypic methods have been assessed using collections of $P$ cepacia, MLEE in our hands has proved to be the most robust and informative typing method available to assess the geographic diversity of $P$ cepacia. Other genotypic techniques that may be descriptive of patient origin include total genomic restriction digest analysis of large or small fragments, classical ribotyping, probe hybridization and nucleotide sequence analysis of portions of the genome. All of these methods have technological advantages or disadvantages, and they have demonstrated sufficient reproducibility to be considered reference genotypic procedures and have established diagnostic merits.

Techniques that have been described for $P$ cepacia and have not yet been validated in studies with adequate scope or about which anecdotal reports in the negative are accumulating, include polymerase chain reaction or 'PCR-ribotyping' (11) and arbitrarily primed amplification fingerprinting (AP-PCR) (12) or RAPD for 'random amplification of polymorphic DNA'. The latter uses short, random single primers in a PCR to generate genomic fingerprints.

The term 'PCR ribotyping' is in itself somewhat misleading in that this technique consists of amplification of the 16S-23S rDNA internal transcribed spacer region using conserved primers. Most strains of $P$ cepacia have multiple spacers that are said to vary in length sufficiently to provide an analysis based on length polymorphisms. We failed to observe adequate amplification length polymorphisms in our study of 83 strains to be enthusiastic about the potential of this technique as a suitable alternative or replacement for classical ribotyping.

From our experience at LCDC with arbitrarily primed PCR fingerprinting of $P$ cepacia, we must agree emphatically with the recent statement by Saunders that "The major drawback of RAPD is its apparent lack of reproducibility as judged by the difficulties experienced by the CPHL (Central Public Health Laboratory) groups who have tried to apply it" (13). We observed variation in both the intensifies and numbers of detectable amplification products when a wide range of random decamers was used as primers, either alone or in combination.

Laboratory evidence confirming person to person spread of $P$ cepacia between $C F$ patients has been primarily based on ribotype data (7-9). Results of our studies suggest that ribotype does indeed reflect an individual strain signature that is valuable for analyzing suspected epidemiological linkages in a setting of the individual clinic or episodes of social contact. Our MLEE data suggest that some clones predominate in wide geographic areas and that some of these may have higher prevalence and mortality. Yet to be determined is whether a higher risk with $P$ cepacia pulmonary infections in $\mathrm{CF}$ patients or a more morbid outcome may be limited to only a few electrophoretic types. These speculations may be confirmed by studies using the repository of strains being collected in Canada under the auspices of the Canadian Cystic Fibrosis Foundation.

In conclusion, development and implementation of discriminatory laboratory typing schemes for $P$ cepacia based on both multilocus electrophoretic type and classical ribotype should lead to the development of meaningful preventive strategies conducive to the best possible quality of life for $\mathrm{CF}$ patients. 


\section{REFERENCES}

1. Isles A, Maclusky I, Corey M, et al. Pseudomonas cepacia infections in cystic fibrosis: An emerging problem. J Pediatr 1984;104:206-10.

2. Tablan OC, Chorba TL, Schidlow DV, et al. Pseudomonas cepacia colonization in patients with cystic fibrosis: Risk factors and clinical outcome. J Pediatr 1985;107:382-7.

3. Statement from the Medical/Scientific Advisory Committee Canadian Cystic Fibrosis Foundation. Epidemiology of Pseudomonas cepacia in cystic fibrosis. Can J Infect Dis 1993;4:163-5.

4. Bingen E, Botzenhart K, Chabanon G, et al. Epidemiology of Pulmonary Infections by Pseudomonas in Patients With Cystic Fibrosis: A Consensus Report. Paris: Association Française de Lutte contre la Mucoviscidose, 1993.

5. Pegues DA, Carson LA, Tablan OC, and the Summer Camp Study Group. Acquisition of Pseudomonas cepacia at summer camps for persons with cystic fibrosis. MMWR $1993 ; 42,456-9$.

6. Thomassen MJ, Demko CA, Doershuk CF, et al. Pseudomonas cepacia: Decrease in colonization in patients with cystic fibrosis. Am Rev Respir Dis 1986:143:669-71.

7. Smith DL, Gumery LB, Smith EG, et al. Epidemic of
Pseudomonas cepacia in an adult cystic fibrosis unit: Evidence of person-to-person transmission. J Clin Microbiol 1993:31;3017-22.

8. Govan JRW, Brown PH, Maddison J, et al. Evidence for transmission of Pseudomonas cepacia by social contact in cystic fibrosis. Lancet 1993:342;15-9.

9. LiPuma JJ, Mortensen JE, Dasen SE, et al, Ribotype analysis of Pseudomonas cepacia from cystic fibrosis treatment centres. J Pediatr 1988:113;859-62.

10. Johnson WM, Tyler SD, Rozee KR. Linkage analysis of geographic and clinical clusters in Pseudomonas cepacia infections by multilocus enzyme electrophoresis (MLEE) and ribotyping. J Clin Microbiol. (In press)

11. Kostman JR, Edlind TD, LiPuma JJ, Stull TL. Molecular epidemiology of Pseudomonas cepacia determined by polymerase chain reaction ribotyping. J Clin Microbiol 1992:30;2084-7.

12. Bingen $\mathrm{EH}$, Weber $\mathrm{M}$, Derelle $\mathrm{J}$, et al. Arbitrarily primed polymerase chain reaction as a rapid method to differentiate crossed from independent Pseudomonas cepacia infections in cystic fibrosis patients. J Clin Microbiol 1993:31;2589-93.

13. Saunders NA. Microbial typing. PHLS Microbiol Dig 1993:10;175-8.

\title{
Immunization in the 90s: Challenges and solutions
}

\author{
October 5 to 7,1994 \\ The Quebec Hilton, Quebec City, Quebec
}

\section{OBJECTIVES}

To present a forum for the discussion and exchange of ideas related to the practical aspects of immunization programs in Canada. The conference will cover issues such as vaccine supply and delivery, multiplication of vaccines and heavier schedules, education, assessment of vaccine programs (vaccine coverage, immunization records, cold chain, surveillance of adverse events), obstacles to immunization, regulations and legislation, and global immunization efforts. Primary focus will be on childhood immunization.

\section{ORGANIZED BY}

The Laboratory Centre for Disease Control, Health Canada, with support from the private sector.

\section{CALL FOR ABSTRACTS}

Time has been allotted within the conference for peer reviewed presentations (poster and oral) that relate to the objectives of the conference. Health units are also encouraged to submit proposals for presentations of material related to education and promotion. Abstract submission forms must be received before June 3, 1994.

To receive a registration package/abstract submission form, contact:

\author{
Mr Chuck Schouwerwou \\ Conference and Committee Coordinator \\ Childhood Immunization Division \\ Bureau of Communicable Disease Epidemiology \\ Laboratory Centre for Disease Control \\ 2nd Floor, LCDC Building \\ Tunney's Pasture, Ottawa, Ontario K1A OL2 \\ Telephone (613) 957-1352, Fax (613) 998-6413
}




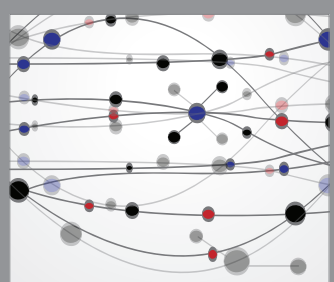

The Scientific World Journal
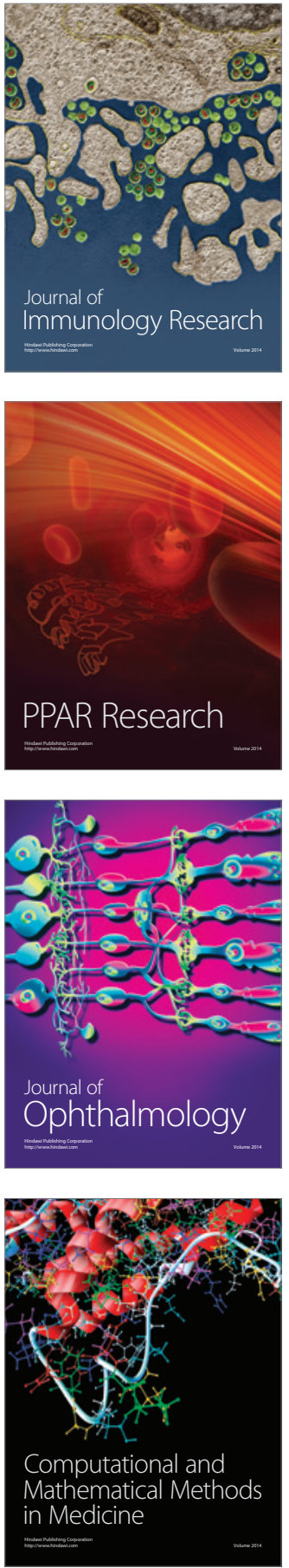

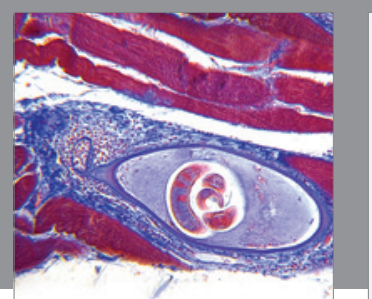

Gastroenterology Research and Practice

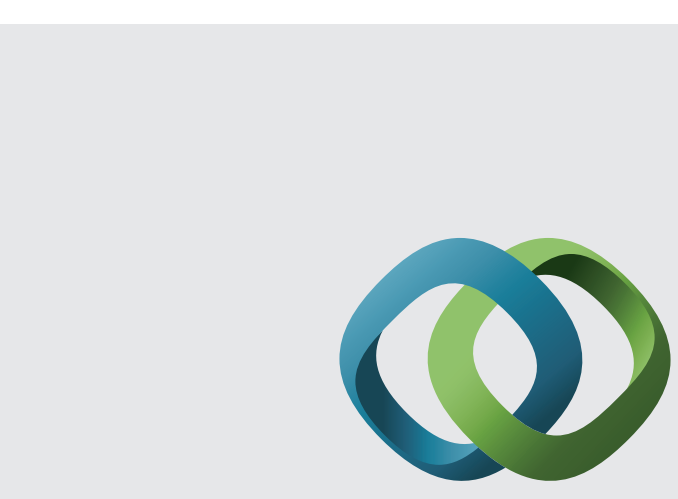

\section{Hindawi}

Submit your manuscripts at

http://www.hindawi.com
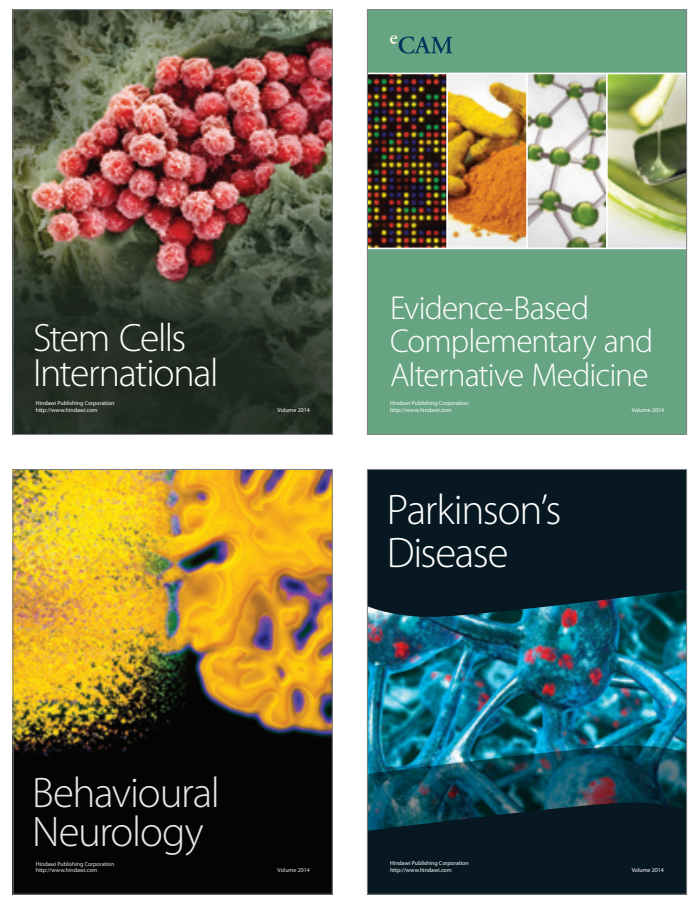
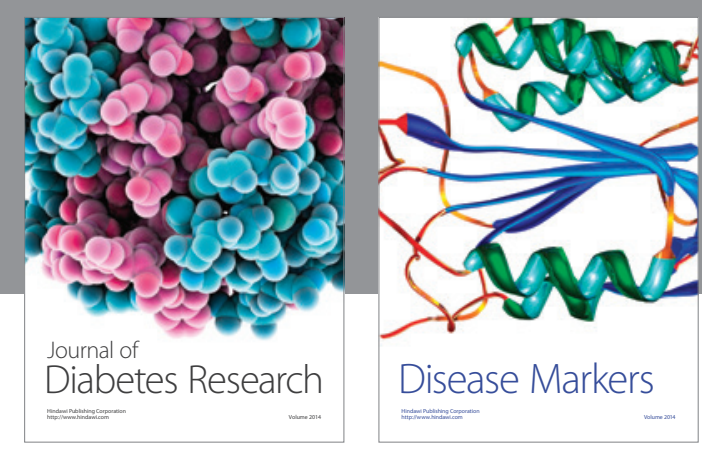

Disease Markers
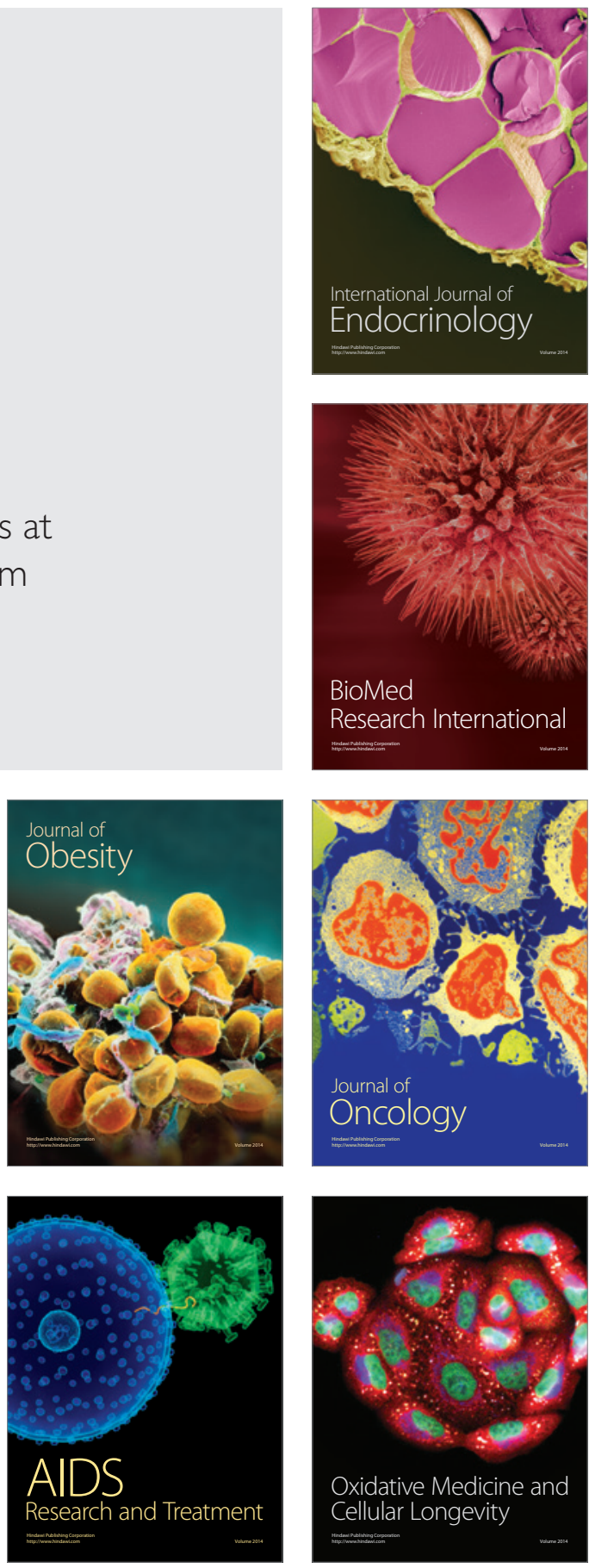